\title{
ANALYSIS OF TUBERCULOSIS DYNAMICAL MODEL WITH DIFFERENT EFFECTS OF TREATMENT
}

\author{
Anindita Henindya Permatasari $1^{*}$, Robertus Heri Soelistyo Utomo ${ }^{2}$ \\ ${ }^{1,2}$ Department of Mathematics, Faculty of Sciences and Mathematics, Diponegoro University, Indonesia \\ Email : ${ }^{1}$ henindya23@gmail.com, ${ }^{2}$ robertusherisoelisty@lecturer.undip.ac.id \\ *Corresponding author
}

\begin{abstract}
A tuberculosis model that integrates pre-infection and active infection stages along with two treatment parameters was studied. The model also considered the death rate due to pre-tuberculosis infection. The basic reproduction ratio was used to investigate the local and global stability of the equilibrium point. The local stability of uninfected equilibrium was analysed using Routh Hurwitz criteria. The existence of endemic equilibrium was given. After we achieved the endemic equilibrium, the global stability of the endemic equilibrium was analyzed using the Lyapunov function. A numerical simulation was studied to illustrate the effect of the treatment on the spread of the tuberculosis disease.
\end{abstract}

Keywords: Tuberculosis, treatment, stability, Routh Hurwitz, Lyapunov

\section{INTRODUCTION}

Tuberculosis is an airborne direct infectious disease. The parts of the body that often affected are the lungs, the kidneys, and spine [1,2]. Tuberculosis already infected a third of the world's population. It was estimated that every year there are around 9 million new tuberculosis patients with 3 million deaths. In developed countries, it is estimated that $75 \%$ of all tuberculosis patients are in the productive age group (aged 15-50 years). Death from tuberculosis disease is $25 \%$ of all preventable deaths [3]. According to WHO, countries in Asia with the highest prevalence are India, China and Indonesia [3,4]. Therefore, we need to work on controlling the spread of tuberculosis.

Tuberculosis is mainly spread from person to person through the droplet containing tuberculosis bacteria that released in the air. There is a difference between being infected with tuberculosis bacteria and having active tuberculosis disease. The stages of tuberculosis are exposed, latent infection (pre infected), and active tuberculosis disease. Early diagnosis and treatment of the person with active tuberculosis disease is important because the stage of tuberculosis cannot be separated from contact with active TB patients. Prevention method such as chemoprophylaxis is also important because it aims to kill or slow the progression of the disease $[5,6]$. Alhough tuberculosis is a treatable infectious disease, it continues to evolve resistance to drugs $[6,7]$.

Mathematical models can provide a useful tool to analyze the dynamics of the spread and control of tuberculosis disease. Some mathematical models for tuberculosis have been developed and studied. Bowong and Tewa [8] formulated a tuberculosis model with SEI type 
and a general contact rate. They derived the equilibrium stability using Lyapunov stability theory. Huo et al. [9] introduced a tuberculosis model with considering tuberculosis patients with the drug sensitive of strain bacterium tuberculosis to be treated. They gave a detailed analysis about positivity and boundedness, existence and uniqueness, also global stability of the equilibrium of the model. Yu et al [10] studied a tuberculosis model with drug resistance cases. They classified the infected classes into tuberculosis infection class and MDR-TB (Multi-Drug Resistance Tuberculosis) infection class, and analysed the local equilibrium stability of the model.

In this study, we established and analysed the dynamical behaviour of the tuberculosis spread by integrating the pre and active infection stages. The model considers two treatment parameters and death rate and it is assumed that there is a death due to pre infection of tuberculosis. Local stability analysis was proved by using Routh Hurwitz criteria, while global stability analysis was proved by using Lyapunov function. We presented a numerical simulations to confirm our results.

\section{MODEL CONSTRUCTION}

We construct the model motivated by Huo and Zou [14]. We consider two kinds of treatments, i.e, treatment for exposed individual and treatment for pre infectious individuals with efficacies $p$ and $r$, respectively. The model consists of five variable and several parameters which is constructed in Figure 1. The model form five nonlinear equations describing populations of susceptible $(S)$, exposed $(E)$, pre infected $\left(I_{1}\right)$, actively infected ( $I_{2}$ ) and recovered $(R)$.

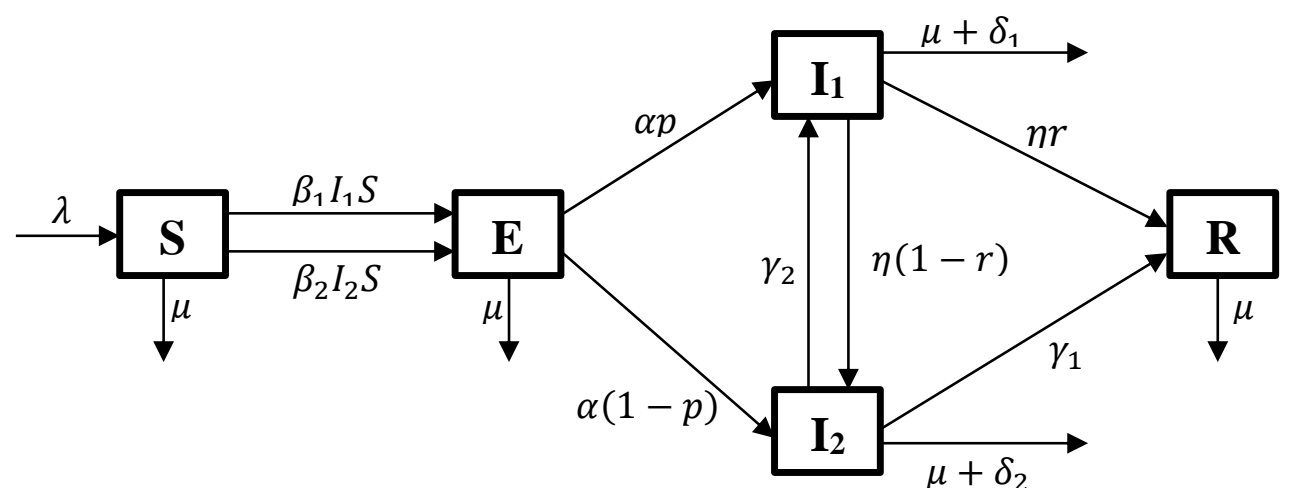

Figure 1. Transfer diagram for tuberculosis model

Parameter $\lambda$ is the recruitment rate of S. The natural mortality rate is represented by $\mu$. Parameter $\beta_{1}$ and $\beta_{2}$ are transmission rate with $I_{1}$ and $I_{2}$ respectively. The first treatment is given to exposed individuals with rate $p$. In some special cases sometimes the immune system is impaired, it fails to overcome and prevent disease. An exposed person who has been given the treatment and rejection occurs in his/her body can go to $I_{1}$ stage at a rate $\alpha p$ or go to $I_{2}$ stage at a rate $\alpha(1-p)$. The rate of change of an individual from the actively infected stage to the pre infected stage is denoted by $\gamma_{2}$. An individual changes from the 
actively infected stage to the recovered stage with rate $\gamma_{1}$. By $\delta_{1}$ and $\delta_{2}$ we denote the death rate due to $I_{1}$ and $I_{2}$, respectively. A pre infected individual is given the second treatment with $r$ as the treatment effectiveness rate. If the treatment is successful then he/she is heading to the recovery stage at a rate $\eta r$. Otherwise, he/she becomes resistant at a rate $\eta(1-r)$. The model is given in the following.

$$
\begin{aligned}
& \frac{d S}{d t}=\lambda-\beta_{1} I_{I} S-\beta_{2} I_{2} S-\mu S \\
& \frac{d E}{d t}=\beta_{1} I_{1} S+\beta_{2} I_{2} S-(\mu+\alpha) E \\
& \frac{d I_{1}}{d t}=\alpha p E-\left(\mu+\delta_{1}+\eta\right) I_{1}+\gamma_{2} I_{2} \\
& \frac{d I_{2}}{d t}=\alpha(1-p) E+\eta(1-r) I_{1}-\left(\mu+\delta_{2}+\gamma_{1}+\gamma_{2}\right) I_{2} \\
& \frac{d R}{d t}=\eta r I_{1}+\gamma_{1} I_{2}-\mu R
\end{aligned}
$$

\section{ANALYSIS OF THE MODEL}

The model $(1)-(5)$ is studied to get the basic reproduction ratio and explore the stability of uninfected state, and stability of endemic equilibrium.

\subsection{Basic Reproduction Ratio}

We derive the ratio $\mathfrak{R}_{0}$ using the next generation matrix. The model (1) - (5) has an uninfected equilibrium point. The uninfected equilibrium point is defined as the point at which there is no disease in the population $\left(I_{1}=I_{2}=0\right)$. By making all right hand side of model (1) - (5) equal to zero and solving the equations, thus, we get the uninfected equilibrium point $U E=\left(\frac{\lambda}{\mu}, 0,0,0,0\right)$. From Dickmann [11], we can obtain the next generation matrix as follows,

$$
K=\left[\begin{array}{ccc}
\Psi_{1} & \Psi_{2} & \Psi_{3} \\
0 & 0 & 0 \\
0 & 0 & 0
\end{array}\right]
$$

where,

$$
\begin{aligned}
& \Psi_{1}=\frac{\lambda \alpha\left(\left(\mu p+\delta_{2} p+\gamma_{1} p+\gamma_{2}\right) \beta_{1}+\left((1-p)\left(\mu+\delta_{1}\right)+(1-p r) \eta\right) \beta_{2}\right)}{\mu(\mu+\alpha)\left[\left(\mu+\delta_{1}+\eta\right)\left(\mu+\delta_{2}+\gamma_{1}\right)+\left(\mu+\delta_{1}+\eta r\right) \gamma_{2}\right]}, \\
& \Psi_{2}=\frac{\left(\mu+\delta_{2}+\gamma_{1}+\gamma_{2}\right) \lambda \beta_{1}+\lambda(1-r) \eta \beta_{2}}{\mu\left[\left(\mu+\delta_{1}+\eta\right)\left(\mu+\delta_{2}+\gamma_{1}\right)+\left(\mu+\delta_{1}+\eta r\right) \gamma_{2}\right]}, \\
& \Psi_{3}=\frac{\lambda \gamma_{2} \beta_{1}+\left(\mu+\delta_{1}+\eta\right) \beta_{2}}{\mu\left[\left(\mu+\delta_{1}+\eta\right)\left(\mu+\delta_{2}+\gamma_{1}\right)+\left(\mu+\delta_{1}+\eta r\right) \gamma_{2}\right]}
\end{aligned}
$$



eigenvalues of matrix $K$

are $0,0, \frac{\lambda \alpha\left[\left(\mu p+\delta_{2} p+\gamma_{1} p+\gamma_{2}\right) \beta_{1}+\left((1-p)\left(\mu+\delta_{1}\right)+(1-p r) \eta\right) \beta_{2}\right]}{\mu(\mu+\alpha)\left[\left(\mu+\delta_{1}+\eta\right)\left(\mu+\delta_{2}+\gamma_{1}\right)+\left(\mu+\delta_{1}+\eta r\right) \gamma_{2}\right]}$. Hence, we have $\Re_{0}$ for system (1) - (5) as follow

$$
\mathfrak{R}_{0}=\frac{\lambda \alpha\left[\left(\mu p+\delta_{2} p+\gamma_{1} p+\gamma_{2}\right) \beta_{1}+\left((1-p)\left(\mu+\delta_{1}\right)+(1-p r) \eta\right) \beta_{2}\right]}{\mu(\mu+\alpha)\left[\left(\mu+\delta_{1}+\eta\right)\left(\mu+\delta_{2}+\gamma_{1}\right)+\left(\mu+\delta_{1}+\eta r\right) \gamma_{2}\right]}
$$

Next, the local stability of equilibrium is presented in the following subsection.

\subsection{Local Stability of Uninfected Equilibrium}

The local stability of the model (1) - (5) can be proved by using linearization RouthHurwitz criterion.

Theorem 1: If $\mathfrak{R}_{0}<1$, the uninfected equilibrium $U E=\left(\frac{\lambda}{\mu}, 0,0,0,0\right)$ is locally asymptotically stable.

Proof: The model (1) - (5) has Jacobian matrix at $U E$ in the following,

$$
J(U E)=\left[\begin{array}{ccccc}
-\mu & 0 & -\beta_{1} \frac{\lambda}{\mu} & -\beta_{2} \frac{\lambda}{\mu} & 0 \\
0 & -(\mu+\alpha) & \beta_{1} \frac{\lambda}{\mu} & \beta_{2} \frac{\lambda}{\mu} & 0 \\
0 & \alpha p & -\left(\mu+\delta_{1}+\eta\right) & \gamma_{2} & 0 \\
0 & \alpha(1-p) & \eta(1-r) & -\left(\mu+\delta_{2}+\gamma_{1}+\gamma_{2}\right) & 0 \\
0 & 0 & \eta r & \gamma_{1} & -\mu
\end{array}\right]
$$

The matrix (8) has two eigenvalues $-\mu$ and other three eigenvalues are the solution of the equation

$$
\zeta^{3}+A_{2} \zeta^{2}+A_{1} \zeta+A_{0}=0
$$

where,

$$
\begin{aligned}
A_{2}= & (\mu+\alpha)+\left(\mu+\delta_{1}+\eta\right)+\left(\mu+\delta_{2}+\gamma_{1}+\gamma_{2}\right), \\
A_{1}= & \frac{\left(p \beta_{1}+(1-p) \beta_{2}\right)(\mu+\alpha)\left(\left(\mu+\delta_{1}+\eta\right)\left(\mu+\delta_{2}+\gamma_{1}\right)+\left(\mu+\delta_{1}+\eta r\right) \gamma_{2}\right)}{\left(\mu p+\delta_{2} p+\gamma_{1} p+\gamma_{2}\right) \beta_{1}+\left((1-p)\left(\mu+\delta_{1}\right)+(1-p r) \eta\right) \beta_{2}}\left(1-\mathfrak{R}_{0}\right)+ \\
& \frac{\Phi_{1} \beta_{1}}{\left(\mu p+\delta_{2} p+\gamma_{1} p+\gamma_{2}\right) \beta_{1}+\left((1-p)\left(\mu+\delta_{1}\right)+(1-p r) \eta\right) \beta_{2}}+ \\
& \frac{\Phi_{2} \beta_{2}}{\left(\mu p+\delta_{2} p+\gamma_{1} p+\gamma_{2}\right) \beta_{1}+\left((1-p)\left(\mu+\delta_{1}\right)+(1-p r) \eta\right) \beta_{2}},
\end{aligned}
$$

with 


$$
\begin{gathered}
\Phi_{1}=\gamma_{2}\left[\eta\left((1+r p)\left(\gamma_{1}+\delta_{2}\right)+(1-r p) \alpha+2 \mu+\gamma_{2} r\right)+(1-p) \delta_{1} \alpha+\mu\left(\mu(1+p)+\delta_{1}+\alpha\right)\right. \\
\left(2 \mu+\delta_{1}+\alpha\right)\left((1+p)\left(\gamma_{1}+\delta_{2}\right)+\mu+\gamma_{2}\right)+p\left(\mu+\gamma_{1}+\delta_{2}\right)^{2}\left(\delta_{1}+\alpha+2 \mu+\eta\right) \\
\Phi_{2}=\left((1-p r) \eta^{2}+(1-p)\left(\mu+\delta_{1}\right)^{2}\right)\left(\gamma_{1}+\delta_{2}+\alpha+2 \mu\right)+\left((1-p r) r \eta^{2}+(1-p)\right. \\
\left.\left(\mu+\delta_{1}\right)^{2}\right) \gamma_{2}\left((p-1) \alpha+(1-p) \delta_{1}\right)\left(\gamma_{1}+\delta_{2}+\gamma_{2} r\right) \eta+(1-p r)\left(\alpha+2 \mu+\delta_{1}\right) \\
\left(\gamma_{1}+\delta_{2}+\gamma_{2}\right) \eta+(1-p)\left((2 \mu+\alpha) \delta_{1}+\mu^{2}\right)+(1-p r)\left(3 \mu^{2}+2 \mu\left(\alpha+\delta_{1}\right)+\alpha \delta_{1}\right) \\
A_{0}=(\mu+\alpha)\left(\left(\mu+\delta_{1}+\eta\right)\left(\mu+\delta_{2}+\gamma_{1}\right)+\left(\mu+\delta_{1}+\eta r\right) \gamma_{2}\right)\left(1-\mathfrak{R}_{0}\right) .
\end{gathered}
$$

Then, we compute $A_{1} A_{2}-A_{0}$

$$
\begin{aligned}
A_{1} A_{2}-A_{0}= & (\mu+\alpha)\left(\left(\mu+\delta_{1}+\eta\right)\left(\mu+\delta_{2}+\gamma_{1}\right)+\left(\mu+\delta_{1}+\eta r\right) \gamma_{2}\right)\left(1-\mathfrak{R}_{0}\right) \\
& {\left[\frac{\left(p\left(2 \mu+\delta_{1}+\eta+\alpha\right)+(p-1) \gamma_{2}\right) \beta_{1}+\left((1-p)\left(2 \mu+\delta_{2}+\gamma_{1}+\gamma_{2}+\alpha\right)+(r-1) p \eta\right) \beta_{2}}{\left(\mu p+\delta_{2} p+\gamma_{1} p+\gamma_{2}\right) \beta_{1}+\left((1-p)\left(\mu+\delta_{1}\right)+(1-p r) \eta\right) \beta_{2}}\right] } \\
& +\frac{A_{2} \Phi_{1} \beta_{1}}{\left(\mu p+\delta_{2} p+\gamma_{1} p+\gamma_{2}\right) \beta_{1}+\left((1-p)\left(\mu+\delta_{1}\right)+(1-p r) \eta\right) \beta_{2}}+ \\
& \frac{A_{2} \Phi_{2} \beta_{2}}{\left(\mu p+\delta_{2} p+\gamma_{1} p+\gamma_{2}\right) \beta_{1}+\left((1-p)\left(\mu+\delta_{1}\right)+(1-p r) \eta\right) \beta_{2}}
\end{aligned}
$$

We see that $A_{1} A_{2}-A_{0}>0$, when $\mathfrak{R}_{0}<1$. Hence, the equilibrium $U E=\left(\frac{\lambda}{\mu}, 0,0,0,0\right)$ is locally asymptotically stable by Routh-Hurwitz criterion. It means, over time the disease will disappear.

\subsection{Global Stability for the Endemic Equilibrium}

The existence of an endemic equilibrium of model (1) - (5) is guaranteed if $\mathfrak{R}_{0}>1$. The model (1) - (5) has endemic equilibrium $E E=\left(S^{*}, E^{*}, I_{1}{ }^{*}, I_{2}{ }^{*}, R^{*}\right)$, where

$$
\begin{aligned}
S^{*} & =\frac{(\mu+\alpha)\left(\left(\mu+\delta_{1}\right)\left(\mu+\delta_{2}+\gamma_{1}+\gamma_{2}\right)+\eta\left(\mu+\delta_{2}+\gamma_{1}+\gamma_{2} r\right)\right)}{\beta_{1} \alpha\left(\mu p+\delta_{2} p+\gamma_{1} p+\gamma_{2}\right)+\beta_{2} \alpha\left(\left(\mu+\delta_{1}+\eta\right)(1-p)+\eta p(1-r)\right)}, \\
E^{*} & =\frac{I_{2}\left(\left(\mu+\delta_{1}\right)\left(\mu+\delta_{2}+\gamma_{1}+\gamma_{2}\right)+\eta\left(\mu+\delta_{2}+\gamma_{1}+\gamma_{2} r\right)\right)}{\alpha\left(\left(\mu+\delta_{1}\right)(1-p)+\eta(1-p r)\right)}, \\
I_{1}^{*}= & \frac{I_{2}\left(p \mu+p \delta_{2}+p \gamma_{1}+\gamma_{2}\right)}{\left(\mu+\delta_{1}\right)(1-p)+\eta(1-p r)}, \\
R^{*}= & \frac{I_{2}\left(\gamma_{1}(1-p)\left(\mu+\delta_{1}\right)+\eta\left(p r \mu+p r \delta_{2}+\gamma_{2} r+\gamma_{1}\right)\right)}{\mu\left(\left(\mu+\delta_{1}\right)(1-p)+\eta(1-p r)\right)} .
\end{aligned}
$$

The solution $I_{2}{ }^{*}$ fulfills the linear equation

$$
a_{1} I_{2}+a_{0}=0
$$


where,

$$
\begin{aligned}
a_{1}= & (\mu+\alpha)\left(\mu p+\delta_{2} p+\gamma_{1} p+\gamma_{2}\right)\left(\left(\mu+\delta_{1}\right)\left(\mu+\delta_{2}+\gamma_{1}+\gamma_{2}\right)+\eta\left(\mu+\delta_{2}+\gamma_{1}+\gamma_{2} r\right)\right) \beta_{1}+ \\
& (\mu+\alpha)\left(\left(\mu+\delta_{1}\right)(1-p)+\eta(1-p r)\right)\left(\left(\mu+\delta_{1}\right)\left(\mu+\delta_{2}+\gamma_{1}+\gamma_{2}\right)+\eta\left(\mu+\delta_{2}+\gamma_{1}+\gamma_{2} r\right)\right) \beta_{2} \\
a_{0}= & \mu(\mu+\alpha)\left(\left(\mu+\delta_{1}\right)(1-p)+\eta(1-p r)\right)\left(\left(\mu+\delta_{1}\right)\left(\mu+\delta_{2}+\gamma_{1}+\gamma_{2}\right)+\eta\left(\mu+\delta_{2}+\gamma_{1}+\gamma_{2} r\right)\right) \\
& \left(1-\mathfrak{R}_{0}\right) .
\end{aligned}
$$

The unique solution $I_{2}{ }^{*}$ of equation (9) is positive if and only if $\frac{a_{0}}{a_{1}}<0$ or $\mathfrak{R}_{0}>1$. Hence, when $\mathfrak{R}_{0}>1$, the endemic equilibrium exists.

Theorem 2: If $\mathfrak{R}_{0}>1$, the endemic equilibrium $E E=\left(S^{*}, E^{*}, I_{1}{ }^{*}, I_{2}{ }^{*}, R^{*}\right)$ of the system $(1$ 5) is globally asymptotically stable.

Proof: Given a candidate Lyapunov function, $F \in C^{1}$, as follows,

$$
\begin{gathered}
F=\left(S-S^{*}-S^{*} \ln \frac{S}{S^{*}}\right)+c_{1}\left(E-E^{*}-E^{*} \ln \frac{E}{E^{*}}\right)+c_{2}\left(I_{1}-I_{1}{ }^{*}-I_{1}{ }^{*} \ln \frac{I_{1}}{I_{1}{ }^{*}}\right) \\
+c_{3}\left(I_{2}-I_{2}{ }^{*}-I_{2}{ }^{*} \ln \frac{I_{2}}{I_{2}^{*}}\right)+c_{4}\left(R-R^{*}-R^{*} \ln \frac{R}{R^{*}}\right)
\end{gathered}
$$

with positive constant $c_{1}, c_{2}, c_{3}, c_{4}$ to be determined. The type of Lyapunov function that we used has been mentioned in [22], [23], [24], [25], [26]. Derivative of $F$ with respect to $t$ is given as follows

$$
\begin{aligned}
\frac{d F}{d t}= & \left(1-\frac{S^{*}}{S}\right) \frac{d S}{d t}+c_{1}\left(1-\frac{E^{*}}{E}\right) \frac{d E}{d t}+c_{2}\left(1-\frac{I_{1}^{*}}{I_{1}^{*}}\right) \frac{d I_{1}^{*}}{d t}+c_{3}\left(1-\frac{I_{2}^{*}}{I_{2}^{*}}\right) \frac{d I_{2}^{*}}{d t}+c_{4}\left(1-\frac{R^{*}}{R}\right) \frac{d R}{d t} \\
= & K-\mu S-\left(\lambda \frac{S^{*}}{S}\right)-\left(c_{1} \beta_{1} I_{1} S \frac{E^{*}}{E}+c_{1} \beta_{2} I_{2} S \frac{E^{*}}{E}\right)-\left(c_{2} \alpha p E \frac{I_{1}^{*}}{I_{1}}+c_{2} \gamma_{2} I_{2} \frac{I_{1}^{*}}{I_{1}}\right) \\
& -\left(c_{3} \alpha(1-p) E \frac{I_{2}^{*}}{I_{2}}+c_{3} \eta(1-r) I_{1} \frac{I_{2}^{*}}{I_{2}}\right)-\left(c_{4} \eta r I_{1} \frac{R^{*}}{R}+c_{4} \gamma_{1} I_{2} \frac{R^{*}}{R}\right) \\
& +\left(c_{2} \alpha p+c_{3} \alpha(1-p)-c_{1}(\mu+\alpha)\right) E+\left(\beta_{1} S^{*}+c_{4} \eta r+c_{3} \eta(1-r)\right. \\
& \left.-c_{2}\left(\mu+\delta_{1}+\eta\right)\right) I_{1}+\left(\beta_{2} S^{*}+c_{2} \gamma_{2}+c_{4} \gamma_{1}-c_{3}\left(\mu+\delta_{2}+\gamma_{1}+\gamma_{2}\right)\right) I_{2}-c_{4} \mu R \\
& +\left(-\beta_{1}+c_{1} \beta_{1}\right) I_{1} S+\left(-\beta_{2}+c_{1} \beta_{2}\right) I_{2} S
\end{aligned}
$$

where $K=\lambda+\mu S^{*}+c_{1}(\mu+\alpha) E^{*}+c_{2}\left(\mu+\delta_{1}+\eta\right) I_{1}^{*}+c_{3}\left(\mu+\delta_{2}+\gamma_{1}+\gamma_{2}\right) I_{2}{ }^{*}+c_{4} \mu R^{*}$. We denote

$x=\frac{S}{S^{*}}, y=\frac{E}{E^{*}}, w=\frac{I_{1}}{I_{1}^{*}}, z=\frac{I_{2}}{I_{2}{ }^{*}}, v=\frac{R}{R^{*}}$. The equation (10) becomes 


$$
\begin{aligned}
\frac{d F}{d t}= & K-\mu S^{*} x-\left(\lambda \frac{1}{x}\right)-\left(c_{1} \beta_{1} I_{1}{ }^{*} S^{*} \frac{x w}{y}+c_{1} \beta_{2} I_{2}{ }^{*} S^{*} \frac{x z}{y}\right)-\left(c_{2} \alpha p E^{*} \frac{y}{w}+c_{2} \gamma_{2} I_{2}{ }^{*} \frac{z}{w}\right) \\
& -\left(c_{3} \alpha(1-p) E^{*} \frac{y}{z}+c_{3} \eta(1-r) I_{1}{ }^{*} \frac{w}{z}\right)-\left(c_{4} \eta r I_{1}{ }^{*} \frac{w}{v}+c_{4} \gamma_{1} I_{2}{ }^{*} \frac{z}{v}\right) \\
& -c_{4} \mu R^{*} v+\left(-\beta_{1}+c_{1} \beta_{1}\right) I_{1}{ }^{*} S^{*} x w+\left(-\beta_{2}+c_{1} \beta_{2}\right) I_{2}{ }^{*} S^{*} x z \\
& +\left(c_{2} \alpha p+c_{3} \alpha(1-p)-c_{1}(\mu+\alpha)\right) E^{*} y+\left(\beta_{1} S^{*}+c_{4} \eta r+c_{3} \eta(1-r)\right. \\
& \left.-c_{2}\left(\mu+\delta_{1}+\eta\right)\right) I_{1}{ }^{*} w+\left(\beta_{2} S^{*}+c_{2} \gamma_{2}+c_{4} \gamma_{1}-c_{3}\left(\mu+\delta_{2}+\gamma_{1}+\gamma_{2}\right)\right) I_{2}{ }^{*} z^{*}
\end{aligned}
$$

Calculating the coefficients of $x w, x z, y, w, z$ are equal to zero, so we get

$c_{1}=1$

$c_{1}(\mu+\alpha)=c_{2} \alpha p c_{1}+c_{3} \alpha(1-p)$

$c_{2}\left(\mu+\delta_{1}+\eta\right)=\beta_{1} S^{*}+c_{4} \eta r+c_{3} \eta(1-r)$

$c_{3}\left(\mu+\delta_{2}+\gamma_{1}+\gamma_{2}\right)=\beta_{2} S^{*}+c_{2} \gamma_{2}+c_{4} \gamma_{1}$

We define a set of the terms, $A=\left\{x, y, w, z, v, x w, x z, \frac{1}{x}, \frac{y}{w}, \frac{z}{w}, \frac{y}{z}, \frac{w}{z}, \frac{w}{v}, \frac{z}{v} \frac{x w}{y}, \frac{x z}{y}\right\}$. The four subsets of $A$ are $\left\{x, \frac{1}{x}\right\},\left\{\frac{1}{x}, \frac{y}{w}, \frac{x w}{y}\right\},\left\{\frac{1}{x}, \frac{y}{z}, \frac{x z}{y}\right\},\left\{\frac{z}{w}, \frac{w}{z}\right\}$. The right hand side in (11) becomes

$$
\begin{aligned}
\frac{d F}{d t}= & b_{1}\left(2-x-\frac{1}{x}\right)+b_{2}\left(3-\frac{1}{x}-\frac{y}{w}-\frac{x w}{y}\right)+b_{3}\left(3-\frac{1}{x}-\frac{y}{z}-\frac{x z}{y}\right)+b_{4}\left(2-\frac{z}{w}-\frac{w}{z}\right) \\
& -c_{4} \mu R^{*} v-c_{4} \eta r I_{1}^{*} \frac{w}{v}-c_{4} \gamma_{1} I_{2}{ }^{*} \frac{z}{v}
\end{aligned}
$$

The constants $b_{1}, b_{2}, b_{3}, b_{4}, c_{4}$ can be obtained by considering the relations as follows

$$
\begin{aligned}
& \lambda=\beta_{1} I_{1}{ }^{*} S^{*}+\beta_{2} I_{2}{ }^{*} S^{*}+\mu S^{*} \\
& (\mu+\alpha) E^{*}=\beta_{1} I_{1}{ }^{*} S^{*}+\beta_{2} I_{2}{ }^{*} S^{*} \\
& \left(\mu+\delta_{1}+\eta\right) I_{1}{ }^{*}=\alpha p E^{*}+\gamma_{2} I_{2}{ }^{*} \\
& \left(\mu+\delta_{2}+\gamma_{1}+\gamma_{2}\right) I_{2}{ }^{*}=\alpha(1-p) E^{*}+\eta(1-r) I_{1}{ }^{*}
\end{aligned}
$$

Equating coefficients in equation (11) and (16), we have

$$
\begin{aligned}
& 2 b_{1}+3 b_{2}+3 b_{3}+2 b_{4}=K \\
& b_{1}=\mu S^{*} \\
& b_{1}+b_{2}+b_{3}=\lambda=\beta_{1} I_{1}^{*} S^{*}+\beta_{2} I_{2}{ }^{*} S^{*}+\mu S^{*} \\
& b_{2}=c_{2} \alpha p E^{*}=c_{1} \beta_{1} I_{1}^{*} S^{*} \\
& b_{3}=c_{3} \alpha(1-p) E^{*}=c_{1} \beta_{2} I_{2}^{*} S^{*} \\
& b_{4}=c_{2} \gamma_{2} I_{2}^{*}=c_{3} \eta(1-r) I_{1}^{*} \\
& c_{1}=1,
\end{aligned}
$$




$$
\begin{aligned}
& c_{2}=\frac{\beta_{1} I_{1}{ }^{*} S^{*}}{\alpha p E^{*}} \\
& c_{3}=\frac{\beta_{2} I_{2}{ }^{*} S^{*}}{\alpha(1-p) E^{*}}
\end{aligned}
$$

Then, one finally has

$$
\begin{aligned}
\frac{d F}{d t}= & \mu S^{*}\left(2-x-\frac{1}{x}\right)+\beta_{1} I_{1}{ }^{*} S^{*}\left(3-\frac{1}{x}-\frac{y}{w}-\frac{x w}{y}\right)+\beta_{2} I_{2}{ }^{*} S^{*}\left(3-\frac{1}{x}-\frac{y}{z}-\frac{x z}{y}\right)+ \\
& \frac{\beta_{1} I_{1}{ }^{*} S^{*}}{\alpha p E^{*}} \gamma_{2} I_{2}{ }^{*}\left(2-\frac{z}{w}-\frac{w}{z}\right)-c_{4} \mu R^{*} v-c_{4} \eta r I_{1}{ }^{*} \frac{w}{v}-c_{4} \gamma_{1} I_{2}{ }^{*} \frac{z}{v} \leq 0 .
\end{aligned}
$$

It is clear that $\frac{d F}{d t}=0$ when $S=S^{*}, E=E^{*}, I_{1}=I_{1}^{*}, I_{2}=I_{2}^{*}, R=R^{*}$, so the maximum invariant set of $\left\{\left(S, E, I_{1}, I_{2}, R\right) \mid \frac{d F}{d t}=0\right\} \quad$ is $E E=\left(S^{*}, E^{*}, I_{1}{ }^{*}, I_{2}{ }^{*}, R^{*}\right)$. We conclude that $E E=\left(S^{*}, E^{*}, I_{1}{ }^{*}, I_{2}{ }^{*}, R^{*}\right)$ is globally asymptotically stable. It means, the disease will stay in the population and maybe it will continue to spread. $\square$

\section{NUMERICAL SIMULATION}

We give a numerical simulation to illustarate the effects of administering drugs to exposed and pre-infected individuals. The simulation of the model (1) - (5) when $\lambda=0.08$ [14], $\mu=0.0101$ [12], $\beta_{1}=0.041, \beta_{2}=0.011, \alpha=0.002, \eta=0.185, \gamma_{1}=0.72$ [14], $p=r=0.5 \gamma_{2}=0.069[14], \delta_{1}=0.2[15], \delta_{2}=0.02$ [8] are presented in the Figure 1. By using that parameter values, we get the value of uninfected equilibrium point $U E=\left(\frac{\lambda}{\mu}, 0,0,0,0\right) \cong(7.2908,0,0,0,0)$.

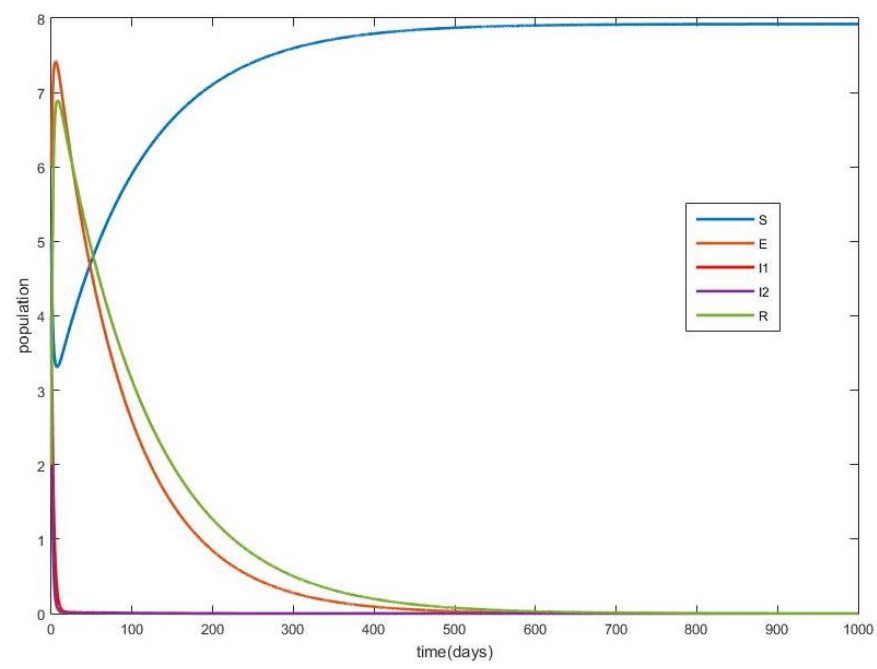

Figure 1: Simulation graph of tuberculosis spread model 
Figure 1 shows that the number of susceptible population decreases in early period and immediately it increases drastically. Tuberculosis starts spreading and increasing in the number of susceptible population due to infection. Either the first treatment or the second treatment has an effect on reducing the number of pre infected and actively infected population. The effectiveness of treatment causing the number of exposed and recovered population increase and going to their equilibrium points. It shows that over time, the population will stable and converges to a value of equilibrium points.

\section{CONCLUSION}

In this study, we considered the dynamics of tuberculosis spread model with two parameters of treatment, that is, treatment for exposed population and treatment for pre infected population. We provided an uninfected equilibrium point and an endemic equilibrium point. Using next generation matrix, we got the basic reproduction ratio. We proved the local stability of uninfected equilibrium and the global stability of endemic equilibrium based on basic reproduction ratio. The uninfected equilibrium is locally asymptotically stable when $\mathfrak{R}_{0}<1$, while the endemic equilibrium is globally asymptotically stable when $\mathfrak{R}_{0}>1$. Treatment plays a crucial role to manage the spread of tuberculosis.

\section{ACKNOWLEDGEMENT}

This work is supported by NONAPBN DPA SUKPA FSM (2021), Diponegoro University, Semarang, Indonesia, under contract number: 2159/UN7.5.8.2/PP/2021

\section{REFERENCES}

[1] Loddenkemper R, Lipman M, Zumla A, "Clinical Aspects of Adult Tuberculosis", Cold Spring Harb Perspect Med, vol. 6, no. 1, 2015.

[2] K.G. Simon, K. Iyawoo, "Management Issue in Tuberculosis in the Asia-Pasific Region", Medical Progress CMP Medica, vol. 32, no.10, pp.486-490, 2005.

[3] World Health Organization, Global Tuberculosis Report 2015, Geneva: World Health Organization, 2015.

[4] World Health Organization, Global Tuberculosis Report 2013, Geneva : World Health Organization, 2013.

[5] US Preventive Services Task Force. "Screening for Latent Tuberculosis Infection in Adults: US Preventive Services Task Force Recommendation Statement". JAMA., vol. 316, no. 9, pp. 962-969, 2016.

[6] H. Getahun, A. Matteelli, R.E.Chaisson, et al, "Latent Mycobacterium Tuberculosis Infection", N Engl J Med, vol. 372, pp. 2127-2135, 2015.

[7] K.J. Seung, S. Keshavjee, M.L. Rich, "Multidrug-Resistant Tuberculosis and Extensively Drug-Resistant Tuberculosis", Cold Spring Harb Perspect Med., vol. 5, no. 9, 2015.

[8] S. Bowong and J.J Tewa, "Global Analysis of a Dynamical Model for Transmission of Tuberculosis with a General Contact Rate", J. Commun. Nonlinear Sci. Numer. Simul., vol. 15, pp. 3621-3631, 2010. 
[9] H.F. Huo, S.J. Dang, Y.N. Li, "Stability of a two-strain tuberculosis model with general contact rate", Abst. Appl. Anal., vol. 2010, pp. 1-31, 2010.

[10] Y. Yu, Y. Shi, and W. Yao, , J. Infect. Dis. Model., vol. 3, pp. 362-372, 2018.

[11] Diekmann, R., and Heesterbeek, JAP., John Wiley and Sons, Chichester, UK, 2000.

[12] S. Bowong and J.J Tewa, "Mathematical Analysis of a Tuberculosis Model with Differential Inefectivity ", J. Commun. Nonlinear Sci. Numer. Simul., vol. 14, pp. 4010-4021, 2009.

[13] H.F. Huo and L.X. Feng, "Global Stability of an Epidemic Model with Incomplete Treatment and Vaccination", Discrete Dyn. Nat. Soc., vol. 2012, pp. 1-14, 2012.

[14] H.F. Huo and M.X. Zou, "Modelling Effects of Treatment at Home on Tuberculosis Transmission Dynamics", J. Appl. Math. Model., vol. 40, pp. 9474-9484, 2016.

[15] P. Rodriguesa, M.G.M. Gomesa, C. Rebelo, "Drug Resistance in Tuberculosis-a Reinfection Model", Theor. Popul. Biol., vol. 71, pp. 196-212, 2007.

[16] R.M.G.J Houben, P.J. Dodd, "The Global Burden of Latent Tuberculosis Infection: A Re-Estimation Using Mathematical Modelling", PLoS Med., vol. 13, 2016.

[17] A. Agyeman, R. Ofori, "Tuberculosis-an overview ", J. Public Health Emerg. , vol. 1, no.7, 2017.

[18] F A Taqiya et al, "Local Stability Analysis for Tuberculosis Epidemic with $\mathrm{SI}_{1} \mathrm{I}_{2} \mathrm{R}$ Model", J. Phys.: Conf. Ser., vol. 1943, 012132, 2021.

[19] N A Lestari et al, "Local Stability Analysis for Tuberculosis Epidemic Model with Different Infection Stages and Treatments", J. Phys.: Conf. Ser., vol. 1943, 012120, 2021.

[20] K.P Wijaya, Sutimin, E. Soewono, and T. Gotz, " On the Existence of a Nontrivial Equilibrium in Relation to the Basic Reproductive Number", Int. J. Appl. Math. Comput. Sci., vol. 27, no. 3, 2017.

[21] Sutimin, R. Herdiana, R.H.S. Utomo, and A.H. Permatasari, "Stability Analysis of Tuberculosis Epidemic Model with Saturated Infection Force ", E3S Web of Conferences, vol. 202, 12023, 2020.

[22] Z. Ma, J. Liu, J. Li, "Stability Analysis for Differential Infectivity Epidemic Models", Nonlinear Analysis RWA, vol. 4, pp. 841-856, 2003.

[23] A. Iggidr, J. Mbang, G. Sallet, J.J. Tewa, "Multi-compartment models", Discrete Contin. Dyn. Syst. Supp., vol. 2007, pp. 506-519, 2007.

[24] C.C. McCluskey, "Lyapunov functions for tuberculosis models with fast and slow progression”, Math. Biosci. Eng., vol. 3, pp. 603-614, 2006.

[25] H. Guo, M.Y. Li, "Global dynamics of a staged progression model for infectious diseases", Math. Biosci. Eng., vol. 3, pp. 513-525, 2006.

[26] J. Liu, T. Zhang, "Global stability for a tuberculosis model”, Math. Comp. Model., vol 54, pp 836-845, 2011. 